\title{
The central Z-disk region of titin is assembled from a novel repeat in variable
} \section{copy numbers}

\author{
Mathias Gautel ${ }^{1, \star}$, David Goulding ${ }^{1}$, Belinda Bullard1, Klaus Weber ${ }^{2}$ and Dieter O. Fürst ${ }^{3}$ \\ ${ }^{1}$ European Molecular Biology Laboratory, Structural Biology Division, Heidelberg, Germany \\ 2Max-Planck-Institut for Biophysical Chemistry, Department of Biochemistry, Göttingen, Germany \\ 3University of Potsdam, Department of Cell Biology, Potsdam, Germany \\ "Author for correspondence
}

\section{SUMMARY}

The giant sarcomeric protein titin (also described as connectin) is composed mainly of immunoglobulin (Ig)like and fibronectin type III (fn3)-like domains arranged consecutively. At both ends of the molecule, these domains are interrupted by sequence insertions. The amino terminus of titin is localized in the Z-disk, a structure of great variability in different muscle types. We have determined the ultrastructural position of sequences in this region of the molecule in skeletal and cardiac muscle by immunoelectron microscopy using antibodies directed against unique epitopes. Titin molecules entering the Z-disk from two half sarcomeres do not significantly overlap, showing that the amino terminus is at the centre of the $Z$-disk. A serine/proline rich site, which can be phosphorylated by kinases in developing muscle tissues, was identified near the amino terminus of titin. Sequence analysis revealed the presence of a novel 45 residue repeat (' $Z$-repeats') in this region of the molecule. The number of titin $Z$-repeats varies due to differential splicing. We propose that this mechanism is a means of assembling Z-disks of variable thickness and mechanical strength.

Key words: Titin, Connectin, Z-disk, Sarcomere, Isoform, Phosphorylation

\section{INTRODUCTION}

The Z-disk of striated muscle is one of the most enigmatic biological structures, owing both to its extreme density and to the insolubility of many of its components. This impedes ultrastructural analysis and makes it difficult to isolate native and undegraded protein constituents. Relatively few proteins are known to localize to the Z-disk (reviewed by Vigoreux, 1994), and the exact position of a number of them is still uncertain. Furthermore, the thickness of Z-disks can vary significantly between different fibre types (Squire, 1981), indicating that the number of proteins incorporated into the structure is regulated in a way that depends upon fibre type.

The assembly of the Z-disk is the starting point of myofibrillogenesis (reviewed by Vigoreaux, 1994; Fürst and Gautel, 1995). The giant muscle protein titin/connectin (Maruyama et al., 1976, 1977; Wang et al., 1979), henceforth called titin, is one of the first proteins appearing in the Z-disk in developing striated muscle (Tokayasu and Maher 1987a,b; Wang et al., 1988; Fürst et al., 1989; Handel et al., 1989; Lin et al., 1994). The integration of titin into nascent sarcomeres begins with the assembly of the amino terminus into the Z-disk and proceeds towards the C-terminal M-band region (Fürst et al., 1989; van der Loop et al., 1996).

It has been proposed that one function of titin may be to act as a molecular blueprint ('molecular ruler') for the layout of the sarcomere (Wang and Wright, 1988; Whiting et al., 1989).
Titin binding to myosin and myosin-associated proteins in the A-band supports this idea (Maruyama et al., 1985; Labeit et al., 1992; Soteriou et al., 1993; Freiburg and Gautel, 1995). However, although the modular array of immunoglobulin-like (Ig) and fibronectin-3-like (fn3) domains in the A-band correlates strikingly with the ultrastructure of the sarcomere (Labeit et al., 1992; Labeit and Kolmerer, 1995; Bennett and Gautel, 1996), the sequence of cardiac titin reveals a particular sequence complexity in both the regions predicted to be in the Z-disk and M-disk (Gautel et al., 1993; Labeit and Kolmerer, 1995; Sebestyén et al., 1995). The identification of sequence insertions in partial rabbit cardiac cDNAs of Z-disk titin, coding for amino acid sequences which can be phosphorylated in vitro by SP-specific protein serine/threonine kinases (Sebestyén et al., 1995) suggests the titin $\mathrm{N}$ terminus may be involved in controlling myofibril assembly. Since the anchorage of titin in the Z-disk is important in assuring its ability to provide an elastic connection between Z-disks and thick filaments (Fürst et al., 1988; Itoh et al., 1988; Whiting et al., 1989; Wang et al., 1993), the structural elements of titin involved in this assembly are of particular interest.

In this work, we have addressed the questions of how the titin sequence relates to the ultrastructure of the Z-disk, which sequence elements may account for the variable thickness of this structure, and whether titin displays further regulatory sequences in the central Z-disk which may be important in controlling its assembly. 


\section{MATERIALS AND METHODS}

\section{Protein expression and purification}

Titin domains were amplified from primary $\lambda$-cDNA clones by PCR as described (Saiki et al., 1985) using specific primers based on the cDNA sequence of human cardiac titin (EMBL data bank X90568). The nomenclature and domain boundaries are as in Labeit and Kolmerer (1995). The cDNA fragments were subcloned into a modified pET vector described previously (Labeit et al., 1992). His6tagged proteins were expressed and purified as described (Politou et al., 1994a,b). Analysis of purified protein was by SDS-PAGE as described (Laemmli, 1970).

\section{cDNA sequencing and analysis}

Fragments from the Z-disk insertion Zis2 (Labeit and Kolmerer, 1995) were amplified from total human cardiac cDNA prepared as described (Labeit and Kolmerer, 1995) by PCR using the primer pair Z/ol1 (5'tttctcgagcCCTGCTAAAAAGACAAAGACAATTG3') and Z/ol2 (5'tttacgegttaCTCCAAAGTGGTCTGCTGAG3'). The fragment pool obtained was subcloned into the modified pET vector (see above), individual clones isolated and grouped by restriction digestion using the flanking $X h o I$ and $M l u I$ sites. Three different length variants could be isolated from human cardiac cDNA, termed ZisS, ZisM and ZisL. DNA sequencing was performed with an automated ALF DNA sequencer (Pharmacia, Sweden) using standard procedures. Sequences have been deposited in the EMBL data library under accession numbers X98114 (ZisS) and X98115 (ZisL).

Sequence prediction and dotplot self-comparison were performed with the UWGCG software package (Devereux et al., 1984). The novel repeat type was identified by sequence self-comparison and by visual inspection, and was used to search the Swiss Prot and EMBL data libraries (release 21) with FASTA or BTFASTA. No significant homologies were found except for the titin sequence itself. Motifs were aligned by GCG-PILEUP and CLUSTAL-W (Thompson et al., 1994) and edited by eye, and homologous positions were boxed with PRETTYPLOT (P. Rice, EMBL).

\section{Antibodies}

The recombinant $\mathrm{Z} 1$ domain and the peptide SPIRMSPARMSPARMSPARM, corresponding to the insertion Zis5, which is located between domains Z6 and Z7 (Labeit and Kolmerer, 1995; Sebestyén et al., 1995) were used for raising rabbit polyclonal antibodies. The peptide was coupled to bovine serum albumin at an estimated molar ratio of 1:10 with bis-succinimidylsuberate following standard protocols (Harlow and Lane, 1988). Chinchilla rabbits were immunized in a standardized scheme with the purified titin domain $\mathrm{Z} 1$ and the peptide-conjugate using titerMax adjuvant (Vaxcel, USA). Antibody production was monitored in test-bleeds by indirect immunofluorescence on cryosections of baboon heart (Bios, Gräfelfing, Germany). For immunohistochemistry, IgG-fractions were affinity-purified on the relevant immobilized antigen as described (Harlow and Lane, 1988).

Monoclonal antibodies (mAbs) were generated by immunizing $\mathrm{Balb} / \mathrm{c}$ mice with native titin prepared from chicken skeletal muscle as described (Fürst et al., 1988, 1989). Fusion of spleen cells with PAI myeloma cells and subsequent selection in HAT medium followed standard protocols (Debus et al., 1983). Resulting antibodies were characterized by western blotting using total muscle extracts and by indirect immunofluorescence on cryosections of skeletal and cardiac muscle samples (Fürst et al., 1988).

Epitope positions were determined in dot-blot assays using the panel of recombinant titin Z-disk fragments described above and I band domains as controls (Politou et al., 1996). Dot blots were performed with $0.1 \mu \mathrm{g}$ of purified protein spotted onto nitrocellulose BA85 membranes (Schleicher \& Schüll, Dassel, Germany). Undiluted overgrown hybridoma supernatants or dilutions of polyclonal sera of
1:1,000 were used to identify reactive domains. Bound antibody was visualized by alkaline-phosphatase-conjugated secondary antibody in a standard colour reaction (Harlow and Lane, 1988). Using total muscle extracts in immunoblotting analysis all the antibodies used in this study were found to be specific for titin. They recognized a single defined epitope in the assay with recombinant titin domains.

\section{Immunoelectron microscopy}

Immunoelectron microscopy was performed essentially as described by Tokayasu (1973) and Fürst et al. (1988). All chemicals were purchased from Sigma or Electron Microscopy Sciences. For cardiac papillary muscle, immunogold labelling was performed on tissue excised from hearts of freshly-killed rabbits on ice and tied immediately under stretch to wooden supports. Specimens were then fixed for 1 hour in rigor solution $\mathrm{A}(0.1 \mathrm{M} \mathrm{KCl}, 20 \mathrm{mM}$ potassium phosphate buffer, pH 6.8, $5 \mathrm{mM} \mathrm{MgCl}_{2}, 5 \mathrm{mM}$ EGTA, $5 \mathrm{mM} \mathrm{NaN}_{3}$ ) with $4 \%$ paraformaldehyde and subsequently dissected into $1 \mathrm{~mm} \times$ $1 \mathrm{~mm} \times 2.5 \mathrm{~mm}$ longitudinal strips. Specimens were cryopreserved in three changes of $2.1 \mathrm{M}$ sucrose, mounted on copper supports and frozen in liquid nitrogen. Sections of $80-120 \mathrm{~nm}$ were cut on a Leica Ultracut $S$ ultramicrotome and collected on carbon-coated grids. First antibody decoration was performed with rabbit polyclonal antibodies at $20 \mu \mathrm{g} / \mathrm{ml}$ of IgG in rigor buffer with $10 \%$ fetal calf serum and 0.02 $\mathrm{M}$ glycine for 30 minutes. After washing of the samples in rigor buffer, bound antibody was visualized by soaking in Protein A coupled to $10 \mathrm{~nm}$ gold particles. The sections were post-fixed with $2.5 \%$ glutaraldehyde for 5 minutes, rinsed briefly in distilled water, stained with $0.3 \%$ uranyl acetate in $2 \%$ methyl cellulose and viewed on a Philips 400T transmission electron microscope. Skeletal muscle (M. psoas) was excised and immunolabelled by soaking in antibody solutions of $0.1-1 \mathrm{mg} / \mathrm{ml}$ as described (Fürst et al., 1988). The immunolabel was subsequently enhanced by soaking in secondary antibody, then embedded in epon and sectioned (Fürst et al., 1988). Electron micrographs were taken at a primary magnification of $\times 10,000$ and $\times 22000$.

\section{Preparation of cell extracts from muscle tissues}

Cardiac left ventricles from adult and neonatal Balb/c mice were dissected from freshly killed animals on ice. Muscle tissue was cut into small fragments, shock frozen in liquid nitrogen and pulverized in a mortar under liquid nitrogen. Homogenized tissue (100 to 150 $\mathrm{mg}$ ) was immediately transferred into ice-cold lysis buffer $(25 \mathrm{mM}$ Hepes, pH 7.2, $100 \mathrm{mM} \mathrm{KCl,} 1 \mathrm{mM}$ EDTA, $1 \mathrm{mM}$ dithiothreitol, 2 $\mu \mathrm{g} / \mathrm{ml}$ leupeptin, $1 \mu \mathrm{M}$ pepstatin, $0.5 \mathrm{mM}$ PMSF, $0.2 \%$ Triton $\mathrm{X}-100)$. Cytosolic and myofibrillar particulate fractions were separated by centrifugation at 3,000 g. Cell fractions were adjusted to $1 \mathrm{mg} / \mathrm{ml}$ total protein (modified Lowry assay, Sigma) by dilution in lysis buffer.

\section{Phosphorylation assays}

Cytosolic fractions and myofibrillar-enriched particulate fractions (2$4 \mu \mathrm{l}$ ) or recombinant cdc2- (New England Biolabs) and ERK-2 (Stratagene) protein kinases were added to phosphorylation assays containing final concentrations of $0.1 \mu \mathrm{g} / \mathrm{ml}$ of the expressed substrate protein in $20 \mu \mathrm{l}$ assay buffer $\left(25 \mathrm{mM}\right.$ Hepes, $\mathrm{pH} 7.2,10 \mathrm{mM} \mathrm{MgCl}_{2}$, $1 \mathrm{mM}$ EGTA, $1 \mathrm{mM}$ DTT, $0.2 \mathrm{mM}$ ATP and $1 \mu \mathrm{Ci}\left[\gamma^{3}{ }^{33} \mathrm{P}\right] \mathrm{ATP}, 3,000$ $\mathrm{Ci} / \mathrm{mM}$; Amersham). Assays were started by addition of a $10 \times \mathrm{ATP}-$ mix and incubated at $37^{\circ} \mathrm{C}$ for 10 minutes. The reactions were stopped by addition of $5 \mu \mathrm{l}$ of sample buffer, heated to $95^{\circ} \mathrm{C}$ for 1 minute and analyzed on $18 \%$ polyacrylamide gels (Laemmli, 1970). Gels were dried and autoradiographed with intensifying screens at $-80^{\circ} \mathrm{C}$ for 12 to 24 hours. For quantitation, radiolabelled protein was precipitated on nitrocellulose filters with 5\% trichloroacetic acid (TCA) and incorporated label measured by liquid scintillation in Ready Safe (Beckman) in a Beckman LS 8100 liquid scintillation counter against background and radionucleotide references for calibration. 


\section{A}

Fig. 1. (A) The modular array of the titin Z-disk region (modified after Sebestyén et al., 1995, and Labeit and Kolmerer, 1995) and the mapped positions of the monoclonal titin antibodies T20, T21 and TM19. The position of T12 is that determined by Labeit and Kolmerer (1995) and according to our own mapping data (not shown). The epitope positions of the newly raised rabbit polyclonal antibodies T-Z1ra and T-SPARMra are also shown, as are the positions of the recombinant protein fragments used in this study. (B) The SPXR-rich regions in the titin Z-disk. Insertion Zis1 contains multiple SPXR consensus sites for ERK- and cdc-like protein kinases (underlined) as well as multiple SP-motifs (italics). Similar motifs are also found in the insertion Zis5 between $\mathrm{Z6}$ and $\mathrm{Z7}$.

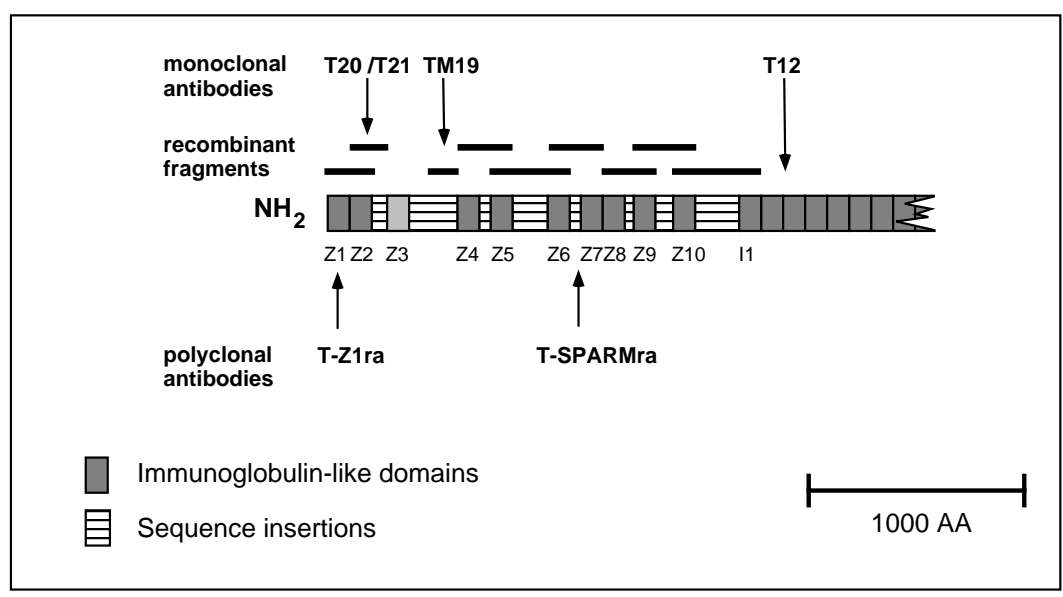

B

Zis1 TPPRIPPKPKSRSPTPPSIAAKAQLARQQSPSPIRH SPSPVRHV

RAP TPSPVRSVSPAARIST SP IRSVRSPLLMRKTQASTVATGPEV

Zis5

\section{RESULTS}

\section{Ultrastructural mapping of the central Z-disk region of titin}

To correlate the sequence information of the Z-disk region of titin with an ultrastructural map of defined epitopes, two approaches were combined: Firstly, a panel of mostly overlapping recombinant fragments of the amino-terminal region $(5.7 \mathrm{~kb}$ in size) of human cardiac titin was generated (Figs 1 and 2). This protein bank was used to map the epitopes of the titin-specific mAbs T20 and T21 described previously. The mAb T20 was assigned to the central Z-disk by immunoelectron microscopy (Fürst et al., 1988). On the panel of recombinant titin fragments mAb T20 bound exclusively to fragments containing the Ig-domain $\mathrm{Z} 2$, thus defining this domain as the epitope (Fig. 3).

The mAb T21 was shown to decorate skeletal sarcomeres $\approx 40 \mathrm{~nm}$ from the Z-disk centre (Fürst et al., 1988); however, on the recombinant domains, it shows the same reactivity as T20, i.e. against constructs at the extreme $\mathrm{N}$ terminus (Fig. 3). At antibody concentrations higher than $10 \mu \mathrm{g} / \mathrm{ml}, \mathrm{T} 21$ cross-reacted weakly with other domains located further downstream (results not shown). Since in the previous immunoelectron microscopy experiments $\operatorname{IgG}$ concentrations of $\sim 1$ $\mathrm{mg} / \mathrm{ml}$ had to be used in order to enhance the antibody decoration by $\mathrm{T} 21$, it can be assumed that the crossreactivity described above resulted in the observed staining at the periphery of Z-disks.

A further titin-specific mAb, TM19, binds close to the Zdisk edge in skeletal muscle (Fig. 4). TM19 binds to the carboxy-terminal fragment Zis $2 \Delta \mathrm{C}$ (cardiac titin residues 550-

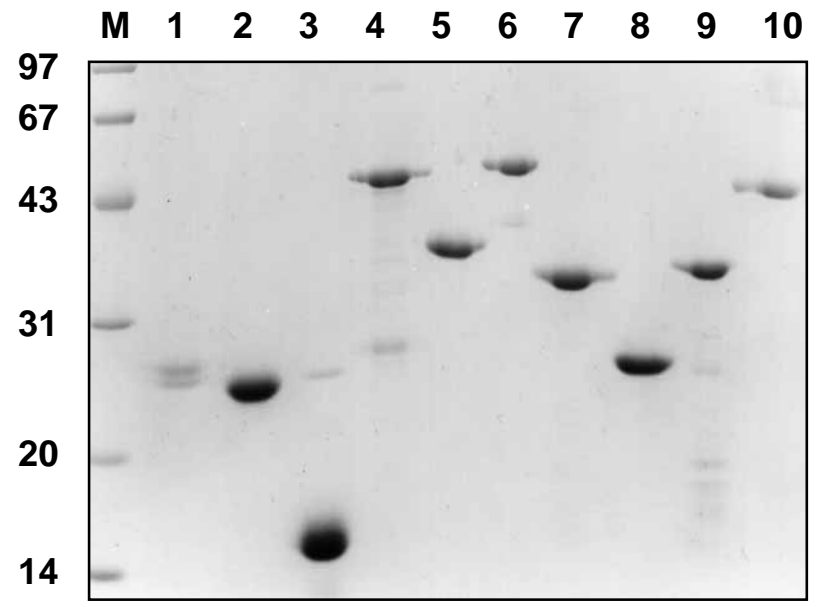

Fig. 2. The recombinant titin Z-disk fragments used to map the monoclonal antibodies T20, T21 and TM19. Purified fragments were analyzed on a $16 \%$ SDS-PAGE gel. Lane 1, Z1-Z2; lane 2, Z2-Zis 1; lane 3, zr3-zr5; lane 4, Zis $2 \Delta \mathrm{C}$; lane 5, Z4-Z5; lane 6, Z5-Z6; lane 7, Z6-Z7; lane 8, Z8-Z9; lane 9, Z9-Z10; lane 10, Z10-I1. Left lane, marker proteins $(97 \mathrm{kDa}$, phosphorylase; $67 \mathrm{kDa}$, bovine serum albumin; $43 \mathrm{kDa}$, ovalbumin; $31 \mathrm{kDa}$, carbonic anhydrase; $20 \mathrm{kDa}$, soybean trypsin inhibitor; $14 \mathrm{kDa}$, lysozyme).

892) but not to the Z-repeats contained in this fragments (Fig. 3). The epitope of TM19 is paraformaldehyde-sensitive, therefore the TM19 localization could not be confirmed in cardiac muscle. These mapping data would imply that the part of titin contained within the dense, central Z-disk is actually rather small. 


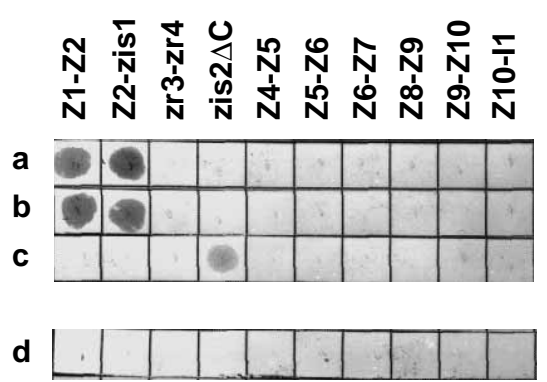

Fig. 3. Determination of the epitope specificities of T20, T21 and TM19 on dot-blots of recombinant titin Z-disk fragments. T20 (a) and T21 (b) bind exclusively to fragments containing the Ig-domain Z2. TM19 (c) binds to the non-modular C-terminal region of Zis2 (Zis $2 \Delta \mathrm{C}$ ). Bottom strip, controls with the goat anti-mouse antibody (d) used for antibody detection.

To confirm and extend the mapping data, two new rabbit polyclonal antibodies were used: the antibody T-Z1ra is directed against the extreme amino-terminal end of titin (cardiac titin residues 1-100), and T-SPARMra is specific for a phosphorylation motif located between Ig-domains 6 and 7 (cardiac titin residues 1,408-1,438; Sebestyén et al., 1955; Labeit and Kolmerer, 1995). T-SPARMra was mapped in skeletal muscle by pre-embedding labelling as described in Materials and Methods. In cardiac muscle, the difficulty in antibody penetrating the very dense Z-disk was circumvented by using cryosections and immunogold labelling. In agreement with the localization of mAb T20 (Fürst et al., 1988), we observed central Z-disk labelling by T-Z1ra using immunogold labelling in cardiac muscle (Fig. 4). In skeletal muscle, T-Z1ra showed dense labelling on the Z-disk (not shown). This independent result unequivocally puts the amino terminus of titin to the centre of the Z-disk. In contrast, T-SPARMra decorated sarcomeres at a position peripheral to Z-disks, $\sim 35 \mathrm{~nm}$ (skeletal muscle) to $\sim 60 \mathrm{~nm}$ (cardiac muscle) from the Z-disk centre (Fig. 4). In skeletal muscle sarcomeres, the fine band of label was at the end of the Z-disk comb (Fig. 4). Interestingly, high concentrations of T-SPARMra dissolved Z-disks (not shown). These ultrastructural findings confirm the epitope mapping data and support the idea of the central Z-disk region of titin spanning mostly between Ig-domains Z1 and Z4.

\section{A second Ser/Pro-rich sequence is revealed in the Z- disk}

The Z-disk region of titin shows a complex pattern of Igdomains with large inter-domain insertions (Sebestyén et al., 1955; Labeit and Kolmerer, 1995), comparable to the M band portion of titin (Gautel et al., 1993). To understand the primary structure of the two Z-disk insertions located between domains Z2 and Z3 (Zis1) and between domains Z3 and Z4 (Zis2; see also Fig. 1B) in more detail, their sequences were analyzed by self-comparison and by visual inspection. Amino-terminal to Z3, we observed a region strikingly rich in serine and proline residues (Fig. 1B). This sequence contains four repeats of the motif SPXR as well as multiple SP sites. The latter have been shown in many proteins to be essential parts of consensus substrate sites for growth factor and cell cycle dependent protein kinases (Songyang et al., 1994). The SPARM-motifs in Zis5 have been shown to
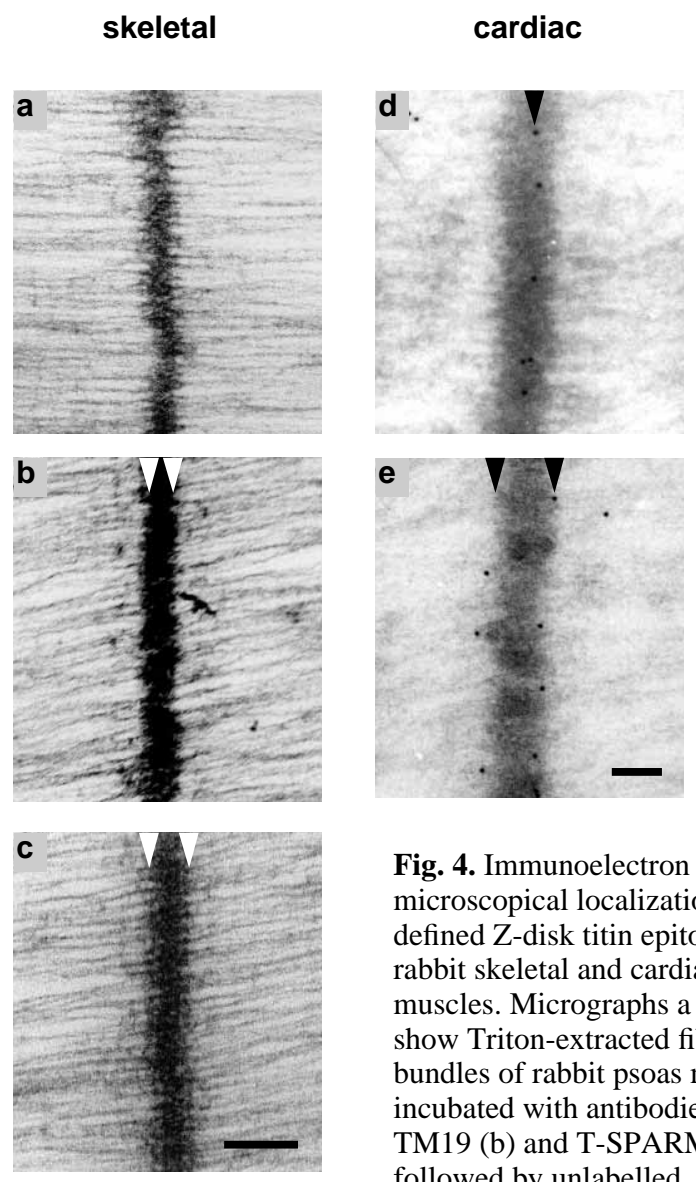

Fig. 4. Immunoelectron microscopical localization of defined Z-disk titin epitopes in rabbit skeletal and cardiac muscles. Micrographs a to $\mathrm{c}$ show Triton-extracted fibre bundles of rabbit psoas muscle incubated with antibodies TM19 (b) and T-SPARMra (c) followed by unlabelled secondary antibody and subsequent processing for electron microscopy. A control sample treated only with secondary antibody is given in a. For epitope localization in cardiomyocytes, cryosections of rabbit heart papillary muscle were treated with T-Z1ra (d) and T-SPARMra (e) and subsequently with Protein A coupled to $10 \mathrm{~nm}$ gold. The positions of antibody decoration are indicated by arrowheads. Note specific labelling of the Z-disk centre and periphery. Bars, $100 \mathrm{~nm}$.

belong to this substrate family (Sebestyén et al., 1995). To test whether this was true for the Zis1 region of titin, the bacterially expressed titin fragment Z2-Z3 was incubated with total muscle extracts from neonatal and adult mice in the presence of $\left[\gamma_{-}{ }^{33} \mathrm{P}\right] \mathrm{ATP}$. Strong phosphate incorporation with extracts from neonatal tissue was observed, indicating that developing muscle most likely contains the kinase activity (Fig. 5A). Since the flanking modules Z2 and Z3 were not found to be phosphorylated (Fig. 5B), we concluded that the insertion Zis1 of titin contains another, previously unidentified, phosphorylation site for Ser/Pro-directed protein kinases in addition to the site in Zis5 (Sebestyén et al., 1955). Cdc2 kinase and ERK-2 kinase were assayed for their ability to phosphorylate Z2-Z3. Fig. 5A demonstrates that both kinases were indeed able to incorporate stoichiometric amounts of phosphate into this titin fragment, with ERK-2 giving rise to the higher levels of phosphate incorporation (about 5 $\mathrm{mol} / \mathrm{mol})$. Similarly, the insertion in Zis5 was found to be phosphorylated by these SP-specific protein kinases (Sebestyén et al., 1995). 
Fig. 5. (A) Phosphorylation of titin Z2-Z3 is downregulated in adult muscle. Extracts of neonatal (lane 1) and adult cardiac tissues (lane 2) contain different levels of protein kinases that phosphorylate Z2-Z3 as analyzed by autoradiography of SDS-polyacrylamide gels. The different intensities suggest a

developmental down-regulation of the SPXR-

directed kinases. Phosphate incorporation into Z2-Z3 is achieved by SP-specific protein kinases like recombinant ERK 2 kinase (lane 3) and cdc2 kinase (lane 4). Position of marker proteins as in Fig. 2 are indicated. (B) The insertion between domains $\mathrm{Z} 2$ and Z3 contains the target site for SP-directed kinases. Z2 (lanes 1 and 4), Z2-Z3 (lanes 2 and 5) and Z3 (lanes 3 and 6) were assayed for phosphorylation by ERK 2 . Only the construct Z2-Z3 is phosphorylated, indicating that Zis 1 contains the target sites. Coomassie-stained SDS-polyacrylamide gel, lanes 1 to 3; autoradiograph of corresponding lanes, lanes 4 to 6 .
A

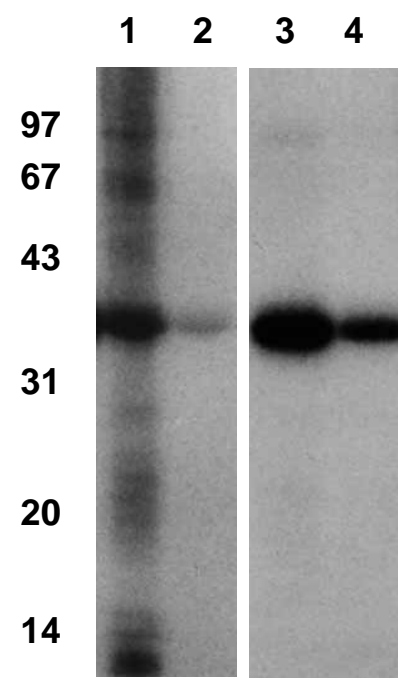

B

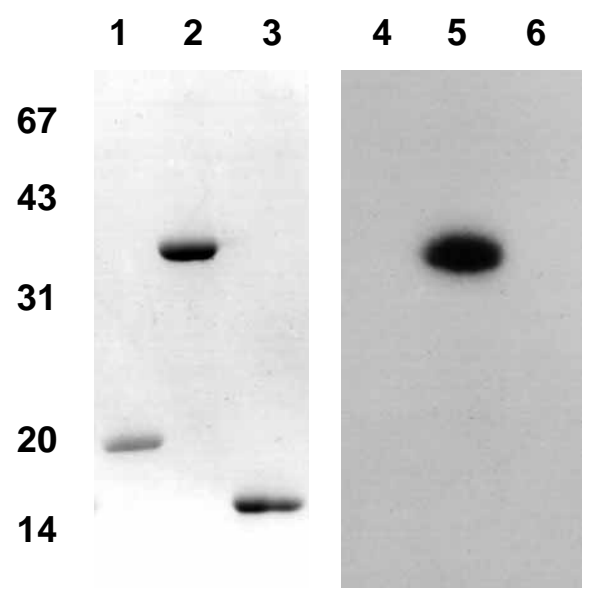

\section{A novel repeat sequence in the titin Z-disk region is expressed in variable copy numbers}

To understand their primary sequence in more detail, the insertions of the cardiac titin Z-disk sequence (EMBL X90568) were analyzed for repetitive sequence elements by computer analysis and visual inspection. In Zis2, we were able to identify the presence of 6 copies of a 45 residue repeat (Fig. 6A). These motifs, which we termed Z-repeats (zr), showed no homology to other proteins in sequence data bases. While the central repeats are essentially identical copies ( $\mathrm{zr} 2, \mathrm{zr} 3, \mathrm{zr} 4)$, the two flanking repeats (zr1 and zr5) are more divergent (Fig. 6B).
Strikingly, these repeats contain hardly any aromatic residues, but are rich in charged and hydrophobic residues arranged in clusters (Fig. 6A).

The immunoelectron microscopical localization of the $\mathrm{T}$ Z1ra, T20, T-SPARMra and TM19 epitopes places the Zrepeats in the part of cardiac titin that is in the centre of the Zdisk. Attempts to isolate the Z-repeat region by PCR from total human cardiac cDNA with the primer pair z/ol1 and z/ol2 (see Materials and Methods) yielded a surprisingly inhomogeneous population of amplified fragments which were further analyzed by DNA sequencing. The schematic representation in Fig. 7

\section{A}
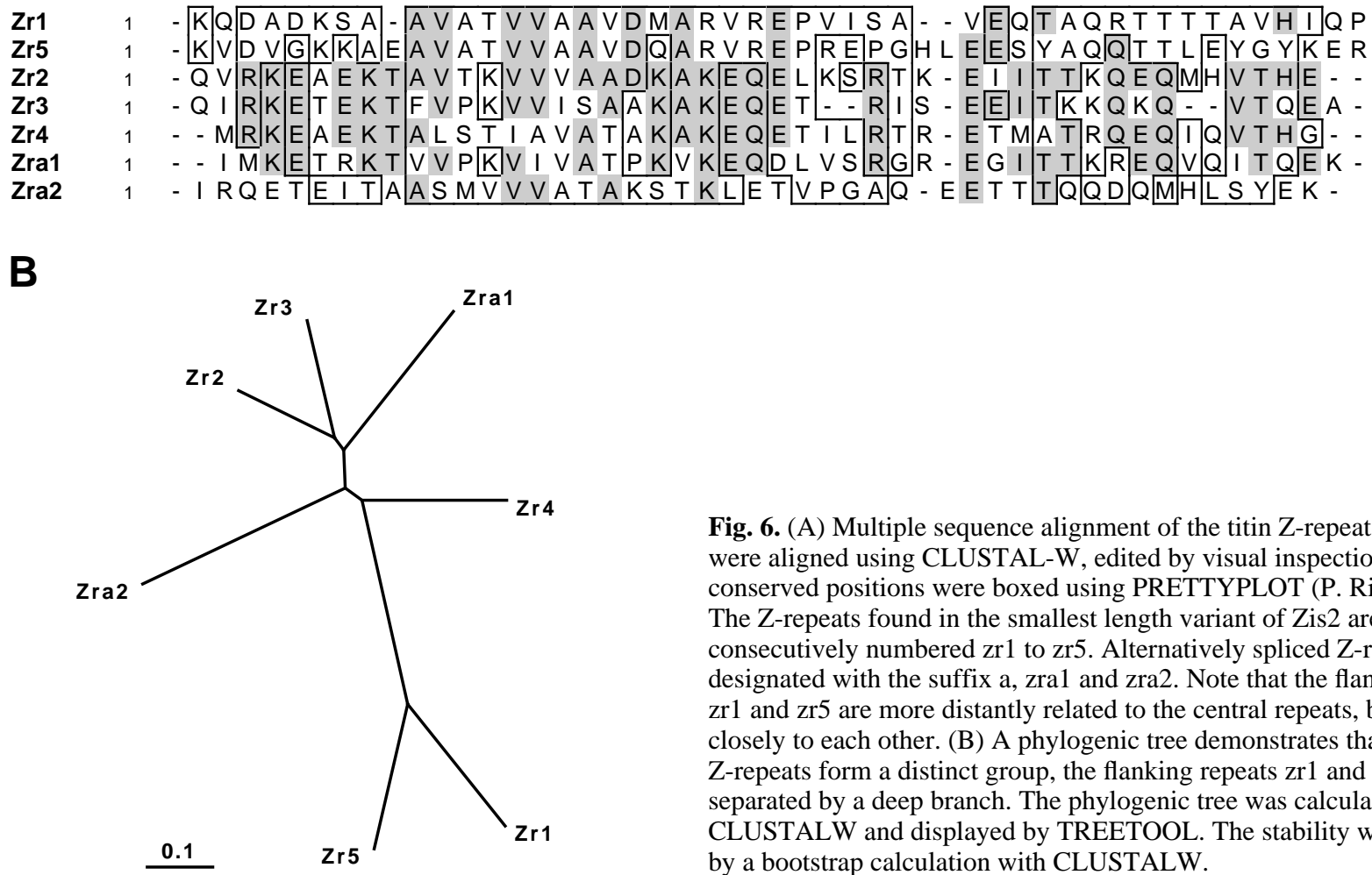

Fig. 6. (A) Multiple sequence alignment of the titin Z-repeats. Motifs were aligned using CLUSTAL-W, edited by visual inspection, and conserved positions were boxed using PRETTYPLOT (P. Rice, EMBL). The Z-repeats found in the smallest length variant of Zis2 are consecutively numbered zr1 to zr5. Alternatively spliced Z-repeats are designated with the suffix a, zra1 and zra2. Note that the flanking repeats zr1 and zr5 are more distantly related to the central repeats, but more closely to each other. (B) A phylogenic tree demonstrates that the central Z-repeats form a distinct group, the flanking repeats $\mathrm{zr} 1$ and $\mathrm{zr} 5$ are separated by a deep branch. The phylogenic tree was calculated by CLUSTALW and displayed by TREETOOL. The stability was checked by a bootstrap calculation with CLUSTALW. 
Fig. 7. A schematic representation of the central Z-disk region of titin defined in this work. The phosphorylation sites in the Z-disk centre and periphery are highlighted. The differentially spliced Zrepeats are marked as a region of variable length. The three splice-variants so far isolated from human cardiac cDNA are shown schematically. The Z-repeats are numbered as in Fig. 6, with the differentially spliced repeats as zra1 and zra2. Note that zra2 is spliced between zr3 and zra1, indicating that expansion of the repeat region occurs by splicing of modules in a carboxy- to amino-terminal order. The Z-repeats in the cardiac titin sequence published previously are marked with $*$. The positions of the antibodies used to map the Z-disk region are shown (grey arrows).

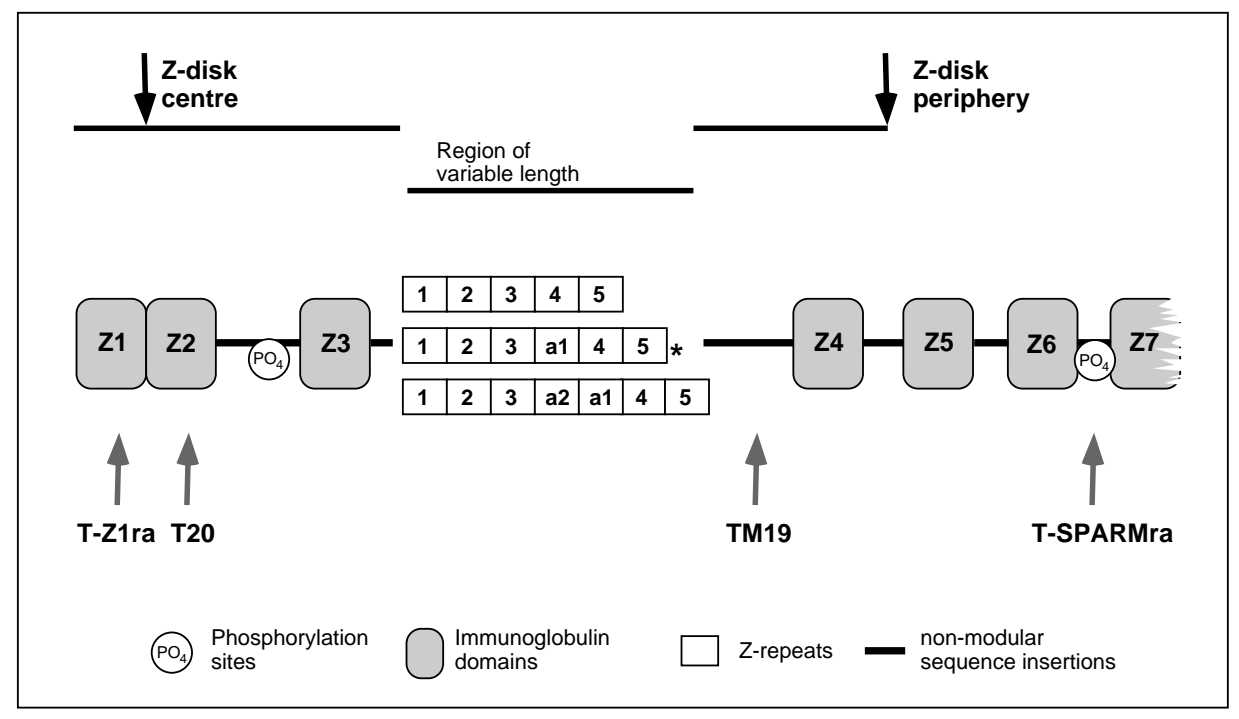

shows that these sequences vary only by the number of central Z-repeats, which can be present in a total of 5, 6 or 7 copies. The smallest form isolated from cardiac cDNA (clone ZisS, X98114) contains the possibly constitutive repeats designated zr1 to zr5. Expansion of this pattern was observed to occur between motifs zr3 and zr4 (clone ZisL, X98115). The differentially spliced repeats (called zra1 and zra2) can be well aligned with the highly homologous group of repeats $\mathrm{zr} 2, \mathrm{zr} 3$ and $\mathrm{zr} 4$ (Fig. 6A) and cluster together in the phylogenic tree (Fig. 6B). These studies thus revealed an unexpectedly complex pattern for cardiac titin with at least three splice variants containing variable numbers of Z-repeats (Fig. 7). The results identify the Z-repeats of titin as a region whose length can be varied by differential splicing and which may therefore be involved in the control of Z-disk thickness and alternative ligand interactions.

\section{DISCUSSION}

To understand in more detail the spatial correlation between distinct titin Z-disk motifs and the sarcomeric ultrastructure of cardiac muscle, we have combined two technical approaches. Firstly, we produced a bank of overlapping recombinant fragments of Z-disk titin to map existing titin monoclonal antibodies of known ultrastructural localization (Fürst et al., 1988) with a resolution of a single Ig domain. Secondly, we raised two sequence-specific polyclonal antibodies and mapped them by immunoelectron microscopy. The central decoration of Zdisks from skeletal muscle by T20 described previously (Fürst et al., 1988) is in good agreement with the decoration pattern of the new polyclonal antibody T-Z1ra in cardiac muscle, which labels the central Z-disk region (Figs 1, 4). The combined antibody data therefore place the titin $\mathrm{N}$ terminus in the centre of the Z-disk, with minimal or no overlap of the molecules entering the structure from two half sarcomeres.

The central Z-disk region of titin is narrowed down further to the stretch between Ig-domains Z1 and Z4 by the positions of the TM19, T-SPARMra and T-Z1ra epitopes. Their labelling patterns demonstrate that titin enters the Z-disk of cardiac muscle up to about Z4, since TM19 obscures the comb-shaped transition zone at the edge of the skeletal muscle Z-disk (Fig. 4). The SPIRM(SPARM $)_{4}$ motif in Zis5 is situated in this comb-shaped transition zone (Fig. 4). A significant portion of the $\mathrm{N}$-terminal region of titin, previously assigned to the Z-disk (Labeit and Kolmerer, 1995), is therefore actually in the I-band between the Z-disk and the T12-epitope, which is about 100 $\mathrm{nm}$ from the Z-disk centre in skeletal muscle (Fürst et al., 1988).

The alignment of the epitopes of T20, TM19 and TSPARMra implies that there is a largely linear arrangement of titin in this region. Since single Ig-domains of titin measure about $4 \mathrm{~nm}$ each (Pfuhl and Pastore, 1995), and there are only 4 such domains in the Z-disk, accounting for $16 \mathrm{~nm}$, the large sequence insertion between domains Z3 and Z4 may account for a significant stretch of the $60 \mathrm{~nm}$ half Z-disk width of titin in cardiac muscle. Sequence analysis of this insertion reveals the presence of a novel, 45 residue repeat module (Fig. 6A), present in 5 to 7 copies (Fig. 7) in human cardiac muscle. The observation that single Z-repeats can be differentially spliced suggests that this novel motif represents a functional domain. Furthermore, the motif boundaries are clearly defined by their splice junctions. The function of these motifs awaits elucidation, but they may represent a class of protein-protein interaction module specific to the Z-disk of titin. However, attempts to detect an interaction between recombinant titin Z-repeats zr3 and $\mathrm{zr} 4$ and radiolabelled $\alpha$-actinin, a putative ligand, were unsuccessful (data not shown). The interaction between titin and $\alpha$-actinin reported previously (Wang and Jeng, 1992; Astier et al., 1993; Tanabe et al., 1994) may therefore be in other motifs, or may be too weak to be detected by using recombinant fragments. The variability in the number of $\mathrm{Z}$ repeats expressed suggests that the variable length of this segment may be involved in determining the thickness of the striated muscle Z-disk, which is known to show great variability (Squire, 1981; Yamaguchi et al., 1985; Vigoreaux 1994). The presence of further length variants due to additional, consecutively spliced Z-repeats cannot be excluded from our current data. This could explain how the T-SPARMra epitope can be between $35 \mathrm{~nm}$ to $60 \mathrm{~nm}$ from the Z-disk centre 
in different muscle types (Fig. 4). However, a full understanding of the possible permutations of this region requires the complete genomic structure of titin.

The N-terminal region of Zis1 consists of a proline-rich region containing 4 SPXR motifs (Fig. 1b) similar to those found in a direct repeat pattern in the insertion Zis5 between Ig-domains Z6 and Z7 (Sebestyén et al., 1995). As with Zis5, in vitro phosphorylation by SP-directed kinases like cdc2 and ERK-2 is observed with the recombinant fragment Z2-Z3 (Fig. 5). Thus, there is a second candidate for a switch in the control of Z-disk assembly by phosphorylation. Based on our ultrastructural investigations, this putative switch is in the central, rather than the peripheral region of the Z-disk. The region harbouring the new SPXR motifs might interact in a phosphorylation-dependent fashion with modules of other proteins which bind to proline-rich sequences. In this context, we note that the SH3 domains of both nebulin and the cardiac mini-nebulin, nebulette, are localized in the Z-disk (Labeit and Kolmerer, 1995; Moncman and Wang, 1995).

The ultrastructural layout of Z-disk titin presented here should now allow correlation of in vitro binding data of other sarcomeric proteins to the sarcomeric ultrastructure. The phosphorylation motifs in Zis1 and Zis5, as well as the novel titin Z-repeats, may present a new view on Z-disk variability.

This work was supported by the Deutsche Forschungsgemeinschaft (Ga405/3-1, Fu339/1-3). We are grateful to Toby Gibson and Annalisa Pastore for stimulating and productive discussions on sequence alignments, and continuous support of this work. We thank the members of the EMBL peptide and oligo synthesis, and DNAsequencing services for their invaluable support. Special thanks to David Stoddard for reliable animal immunization.

\section{REFERENCES}

Astier, C., Labbé, J.-P., Roustan, C. and Benyamin, Y. (1993). Effects of different enzymatic treatments on the release of titin fragments from rabbit skeletal myofibrils. Biochem. J. 290, 731-734.

Bennett, P. M. and Gautel, M. (1996). Titin domain patterns correlate with the axial disposition of myosin at the end of the thick filament. J. Mol. Biol. 259, 896-903.

Debus, E., Weber, K. and Osborn, M. (1983). Monoclonal antibodies to desmin, the muscle-specific intermediate filament protein. EMBO J. 2, 23052312.

Devereux, J., Haeberli, P. and Smithies O. (1984). A comprehensive set of sequence analysis programs for the VAX. Nucl. Acids Res. 12, 387-395.

Freiburg, A. and Gautel, M. (1995). A molecular map of the interactions of titin and myosin-binding protein C: implications for sarcomeric assembly in familial hypertrophic cardiomyopathy. Eur. J. Biochem. 235, 317-323.

Fürst, D. O., Osborn, M., Nave, R. and Weber, K. (1988). The organization of titin filaments in the half-sarcomere revealed by monoclonal antibodies in immunoelectron microscopy: a map of ten nonrepetitive epitopes starting at the $\mathrm{Z}$ line extends close to the M-line. J. Cell Biol. 106, 1563-1572.

Fürst, D. O., Osborn, M. and Weber, K. (1989). Myogenesis in the mouse embryo: differential onset of expression of myogenic proteins and the involvement of titin in myofibril assembly. J. Cell Biol. 109, 517-527.

Fürst, D. O. and Gautel, M. (1995). The anatomy of a molecular giant: how the sarcomeric cytoskeleton is assembled from molecules of the immunoglobulin superfamily. J. Mol. Cell. Cardiol. 27, 951-959.

Gautel, M., Leonard, K. and Labeit, S. (1993). Phosphorylation of KSPmotifs in the C-terminal region of titin in differentiating myoblasts. EMBO J. 12, 3827-3834

Handel, S. E., Wang, S.-M., Greaser M. L., Schultz, E., Bulinski, J. C. and Lessard, J. L. (1989). Skeletal muscle myofibrillogenesis as revealed with a monoclonal antibody to titin in combination with detection of the $\alpha$ - and $\gamma$ isoforms of actin. Dev. Biol. 132, 35-44.
Harlow, E. and Lane, D. (1988). Monoclonal antibodies. Antibodies: A Laboratory Manual, pp. 174-238. Cold Spring Harbor Laboratory Press, Cold Spring Harbor, NY.

Itoh, Y., Suzuki, T., Kimura, S., Ohashi, K., Higushi, H., Sawada, H., Shimizu, T., Shibata, M. and Maruyama, K. (1988). Extensible and less extensible domains of connectin filaments in stretched vertebrate skeletal muscle sarcomeres as detected by immunofluorescence and immunoelectronmicroscopy using monoclonal antibodies. J. Biochem. 104, 504-508.

Labeit, S., Gautel, M., Lakey, A. and Trinick, J. (1992). Towards a molecular understanding of titin. EMBO J. 11, 1711-1716.

Labeit, S. and Kolmerer, B. (1995). Titins, giant molecules in charge of muscle ultrastructure and elasticity. Science 270, 293-296.

Labeit, S. and Kolmerer, B. (1995). The complete primary structure of human nebulin and its correlation to muscle structure. J. Mol. Biol. 248, 308-315.

Laemmli, U. K. (1970). Cleavage of structural proteins during the assembly of the head of bacteriophage T4. Nature 227, 680-685.

Lin, Z., Lu, M. H., Schultheiss, T., Choi, J., Holtzer, S., DiLullo, C., Fischman, D. A. and Holtzer, H. (1994). Sequential appearance of musclespecific proteins in myoblasts as a function of time after cell division: evidence for a conserved myoblast differentiation program in skeletal muscle. Cell Motil. Cytoskel. 29, 1-19.

Maruyama, K., Natori, R. and Nonomura, Y. (1976). New elastic protein from muscle. Nature 262, 58-59.

Maruyama, K., Matsubara, S., Natori, R., Nonomura, Y., Kimura, S., Ohashi, K., Murakami, F., Handa, S. and Eguchi, G. (1977). Connectin, an elastic protein of muscle: characterization and function. J. Biochem. 82, 317-337.

Maruyama, K., Kimura, S., Yamamoto, K., Wakabayashi, T. and Suzuki, T. (1985). Connectin causes aggregation of myosin rods but not myosin heads. Biomed. Res. 6, 423-427

Moncman, C. L. and Wang, K. (1995). Nebulette: a 107 kD nebulin-like protein in cardiac muscle. Cell Motil. Cytoskel. 32, 205-225.

Pfuhl, M. and Pastore, A. (1995). Tertiary structure of an immunoglobulinlike domain from the giant muscle protein titin: a new member of the I-set. Structure 3, 391-401.

Politou, A., Gautel, M., Pfuhl, M., Labeit, S. and Pastore, A. (1994a). Immunoglobulin-type domains of titin: same fold, different stability? Biochemistry 33, 4730-4737.

Politou, A., Gautel, M., Joseph, C. and Pastore, A. (1994b). Immunoglobulin domains of titin are stabilized by N-terminal extension. FEBS Lett. 352, $27-$ 31.

Politou, A. S., Gautel, M., Improta, S., Vangelisia, L. and Pastore, A. (1996). The elastic I-band region of titin is assembled in a 'modular' fashion by weakly interacting Ig-like domains. J. Mol. Biol. 255, 604-616.

Saiki, R. K., Scharf, S. J., Faloona, F., Mullis, G. T. and Erlich, H. A. (1985). Enzymatic amplification of beta-globin genomic sequences and restriction site analysis for diagnosis of sickle cell anemia. Science 230, 1350-1354.

Sebestyén, M. G., Wolff, J. A. and Greaser, M. L. (1995). Characterization of a $5.4 \mathrm{~kb}$ cDNA fragment from the Z-line region of rabbit cardiac titin reveals phosphorylation sites for proline-directed kinases. J. Cell Sci. 108, 30293027.

Songyang, Z., Blechner, S., Hoagland, N., Hoekstra, M. F., PiwnicaWorms, H. and Cantley, L. C. (1994). Use of an oriented peptide library to determine the optimal substrates of protein kinases. Curr. Biol. 4, 973-982.

Soteriou, A., Gamage, M. and Trinick, J. (1993). A survey of interactions made by the giant protein titin. J. Cell Sci. 104, 119-123.

Squire, J. (1981). Structure of the Z-band. The Structural Basis of Muscular Contraction. pp. 364-375. Plenum Press, New York and London.

Tanabe, R., Tatsumi, R. and Takahashi, K. (1994). Purification and characterization of the $1200-\mathrm{kDa}$ subfragment of connectin filaments produced by $0.1 \mathrm{mM}$ calcium ions. J. Biochem. 115, 351-355.

Tokayasu K. T. (1973). A technique for ultracryotomy of cell suspensions and tissues. J. Cell Biol. 57, 551-565.

Tokayasu, K. T. and Maher P. A. (1987a). Immunocytochemical studies of cardiac myofibrillogenesis in early chick embryos. I. Presence of immunofluorescent titin spots in premyofibril stages. J. Cell Biol. 105, 27812793.

Tokayasu, K. T. and Maher P. A. (1987b). Immunocytochemical studies of cardiac myofibrillogenesis in early chick embryos. II. Generation of $\alpha$ actinin dots within titin spots at the time of the first myofibril formation. $J$. Cell Biol. 105, 2781-2793.

Thompson, J. D., Higgins, D. G. and Gibson, T. J. (1994). CLUSTAL-W: improving the sensitivity of progressive, multiple sequence alignment 
through sequence weighting, position-specific gap penalties and weight matrix choice. Nucl. Acids Res. 22, 4673-4680.

Vigoreaux, J. O. (1994). The muscle Z-band: lessons in stress management. $J$. Muscle Res. Cell Motil. 15, 237-255.

van der Loop, F. T. L., van der Ven, P. F. M., Fürst, D. O., Gautel, M., van Eys, G. J. J. M. and Ramaekers, F. C. S. (1996). Integration of titin into the sarcomeres of cultured differentiating human skeletal muscle cells. Eur. J. Cell Biol. 69, 301-307

Wang, K., McClure, J. and Tu, A. (1979). Titin: major myofibrillar components of striated muscle. Proc. Nat. Acad. Sci. USA 76, 3698-3702.

Wang, K. and Wright, J. (1988). Architecture of the sarcomeric matrix of skeletal muscle: immunoelectron microscopic evidence that suggests a set of parallel inextensible nebulin filaments anchored at the Z line. J. Cell Biol. 107, 2199-2212.

Wang, K., McCarter, R., Wright, J., Beverly, J. and Ramirez-Mitchell, R. (1993). Viscoelasticity of the sarcomere matrix of skeletal muscle - the titin- myosin composite filament is a dual stage molecular spring. Biophys. J. 64 , 1161-1177.

Wang, S.-M. and Jeng, C.-J. (1992). Interaction between titin and $\alpha$-actinin. Biomed. Res. 13, 197-202.

Wang, S.-M., Greaser, M. L., Schultz, E., Bulinski, J. C., Lin, J. J.-C. and Lessard, J. L. (1988). Studies on cardiac myofibrillogenesis with antibodies to titin, actin, tropomosin, and myosin. J. Cell Biol. 107, 1075-1083.

Whiting, A., Wardale, J. and Trinick, J. (1989). Does titin regulate the length of muscle thick filaments? J. Mol. Biol. 205, 263-268.

Yamaguchi, M., Izumimoto, M., Robson, R. M. and Stromer, M. H. (1985), Fine structure of wide and narrow vertebrate muscle Z-lines. A proposed model and computer simulation of Z-line architecture. J. Mol. Biol. 184, 621644.

(Received 23 May 1996 - Accepted 12 August 1996) 